\title{
Potential biomarkers and antagonists for fluoranthene-induced cellular toxicity of bone marrow derived mesenchymal stem cells
}

\author{
Md Moinul Hoque ${ }^{1,2}$, Young Eun Lee ${ }^{1,2}$, Hye Ran Kim ${ }^{3}$, Myung Geun Shin ${ }^{1,2}$ \\ ${ }^{1}$ Department of Laboratory Medicine and Mitochondrial Research Laboratory, ${ }^{2}$ Brain Korea 21 Plus Program, Chonnam National \\ University Medical School, Chonnam National University Hwasun Hospital, Hwasun, ${ }^{3}$ College of Korean Medicine, Dongshin \\ University, Naju, Korea
}

p-ISSN 2287-979X / e-ISSN 2288-0011 https://doi.org/10.5045/br.2019.54.4.253 Blood Res 2019;54:253-261.

Received on August 5, 2019

Revised on September 6, 2019

Accepted on September 7, 2019
Background

Fluoranthene (FR) is a common environmental pollutant that exists in a complex mixture with other polycyclic aromatic hydrocarbons (PAHs). We identified biomarkers for monitoring FR exposure and investigated the rescue effect of FR-induced cellular toxicity via aryl hydrocarbon receptor (AHR) antagonist activity in bone marrow derived mesenchymal stem cells (BM-MSCs).

\section{Methods}

Morphological changes, viability, and rescue effects of an AHR antagonist ( $\mathrm{CH} 223191)$ were examined in BM-MSCs after exposure to FR. Cytotoxic effects were assayed using the tetrazolium-based colorimetric assay. Apoptosis was measured by annexin $\mathrm{V}$ and propidium iodide dye-based flowcytometry assay, mitochondrial membrane potential assay, and nuclear DNA fragmentation assay. Molecular signaling pathways of apoptosis and autophagy were investigated using immunoblotting. Proteomics were performed in order to reveal the spectra of cellular damage and identify biomarkers for FR exposure.

\section{Results}

Exposing BM-MSCs to $\mathrm{FR}\left(\mathrm{IC}_{50}=50 \mu \mathrm{M}\right)$ induced cell death and morphological changes, while the AHR antagonist showed rescue effects. Autophagy was activated and mitochondrial membrane potential was decreased. Proteomic analysis identified 48 deregulated proteins (26 upregulated and 22 downregulated). Among them, annexin A6, pyruvate kinase, UDP-glucose dehydrogenase, and phospholipase A2 could be potential biomarkers for FR exposure.

\section{Conclusion}

The exposure of BM-MSCs to FR induced remarkable alterations in cellular biology and the proteome, allowing for identification of novel biomarkers for FR exposure. Furthermore, AHR antagonists might be able to prevent cellular damage due to FR exposure.

Key Words Fluoranthene, Biomarker, Aryl hydrocarbon receptor antagonist

\section{INTRODUCTION}

Polycyclic aromatic hydrocarbons (PAHs) are common environmental pollutants formed from the incomplete combustion of fossil fuels and are found in the environments in a complex mixture [1]. In addition to being produced naturally by volcanoes and forest fires, most PAHs are released into the environment by man-made processes, including burning fuels such as coals, oils, petroleum, and diesels in vehicle and aircraft exhausts [2, 3]. There are approximately 100 known PAHs which exist in our environment [4]. Once released into the environment, they usually enter our bodies through inhalation, ingestion, and dermal contact in both occupational and non-occupational settings.

Inside our body, they are either metabolized or excreted in urine, bile, and breast milk (occasionally) and are often stored in adipose tissue to a limited extent. PAHs differ with respect to distribution patterns and lipophilic properties [5]. Their lipophilic features enable them to accumulate in 
adipose tissue and breast milk. Urinary and biliary excretion of PAHs is relatively well-organized because of the extensive distribution of enzymes that transform PAHs into intermediate metabolites such as diol-epoxides, reactive redox active o-quinones, and radical cations all of which can react with DNA to form DNA adducts and also alter lipids and proteins. PAHs are predominantly metabolized via CYP1A1 enzymes (enzymes in the P-450 mixed-function oxidase sys- tem) in the liver [6, 7]. Several studies have suggested that certain PAHs are associated with the risk of childhood leukemia, including parental occupational exposure to vehicle exhaust $[8,9]$, paternal smoking $[10,11]$, and proximity to traffic $[12,13]$. The aryl hydrocarbon receptor (AHR) is a member of the family of basic helix-loop-helix transcription factors. AHR binds several exogenous ligands such as natural plant flavonoids, polyphenolics and indoles, as well as PAHs,

A

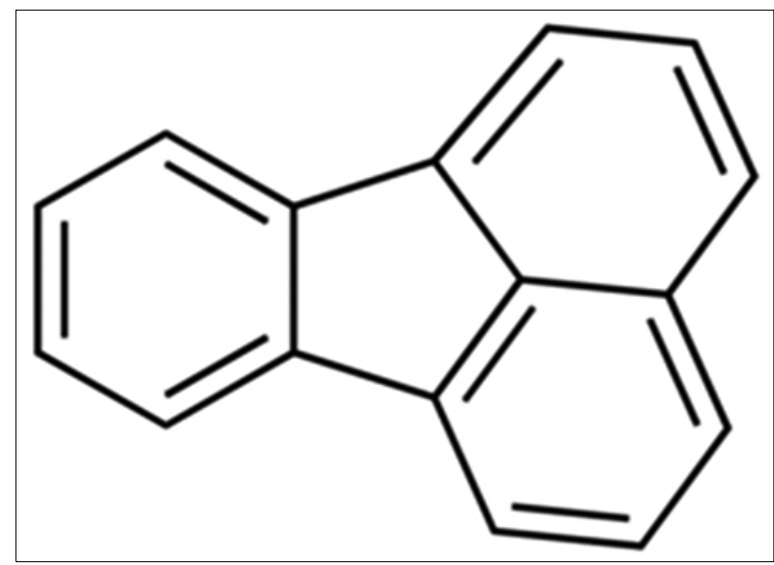

B

Day 1
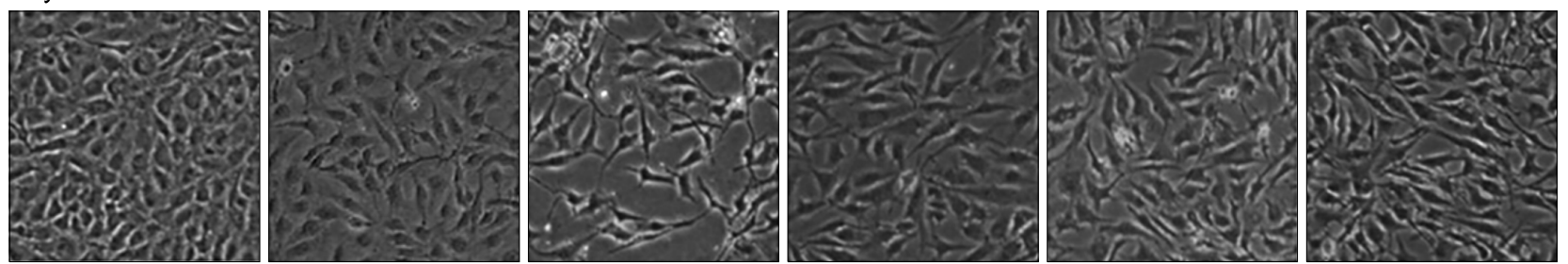

Day 2
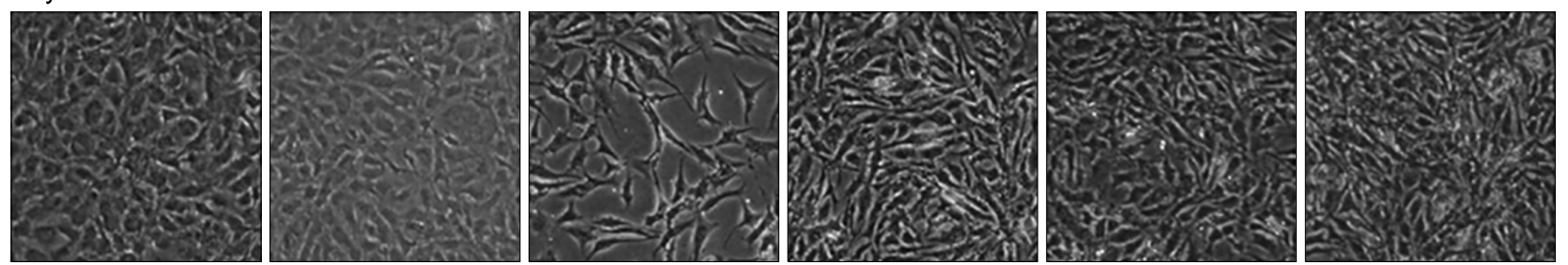

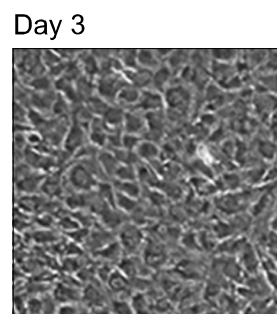

Untreated

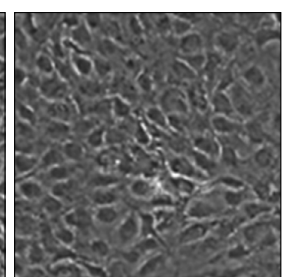

DMSO

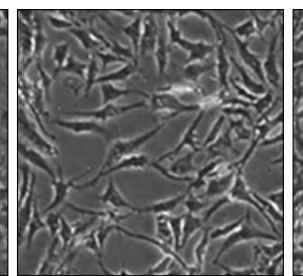

FR $150 \mu \mathrm{M}$

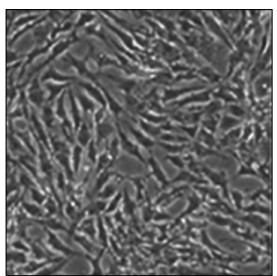

FR $150 \mu \mathrm{m}$

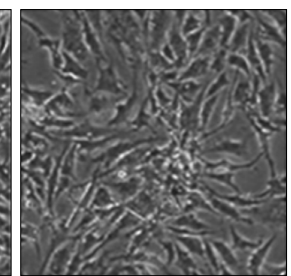

FR $150 \mu \mathrm{m}$

$\mathrm{CH} 223191250 \mathrm{~nm}$

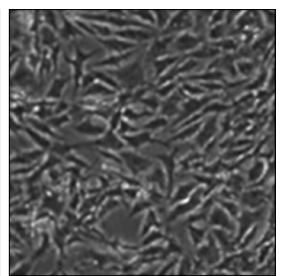

FR $150 \mu \mathrm{m}$ $+$

$\mathrm{CH} 223191100 \mathrm{~nm}$

CH223191500 nm

Fig. 1. Chemical structure of FR and morphological changes of BM-MSCs upon exposure to FR and subsequent antagonistic effects of CH 223191 every 24 hours for up to 72 hours. FR is a polycyclic aromatic hydrocarbon (PAH) that is formed by the fusion of naphthalene and benzene connected by a five-membered ring (A). Morphological changes of BM-MSCs after FR exposure revealed that cells were elongated, detached from the subsurface, and had loosened cell-to-cell attachments. These morphological alterations were partially rescued after co-treatment with an AHR antagonist (CH223191) in a dose-dependent manner (B). 
and dioxin-like compounds. Upon ligand binding to chemicals such as 2,3,7,8-tetrachlorodibenzo-p-dioxin (TCDD), the chaperones dissociate resulting in AHR translocating into the nucleus and dimerizing with AHR nuclear translocator, leading to changes in gene transcription (https:// en.wikipedia.org).

Fluoranthene (FR) is an important PAH candidate that is formed by the fusion of naphthalene and benzene connected by a five-membered ring (Fig. 1A). It is a structural isomer of the PAH candidate pyrene but it is not as thermodynamically stable as pyrene. FR exists in many combustion products with other $\mathrm{PAH}$ candidates and its occurrence is an indicator of low-temperature combustion.

The U.S. Environmental Protection Agency defined FR as one of the 16 priority pollutant PAHs. It has been classified by the International Agency for Research on Cancer as a group 3 carcinogen, i.e. "not classifiable as to its carcinogenicity to humans" [14]. However, it was found to possess carcinogenic properties in new-born mice according to short-term lung tumor assays [15].

Mitochondria are maternally inherited and are the main source of ATP generation in cells. Several studies in mammalian models indicate that PAH toxicity mainly targets the mitochondria within the cell. These studies have shown that PAHs concentrate in the mitochondria and are associated with reduced ATP production [16], loss of mitochondrial membrane potential, changes in mitochondrial morphology $[17,18]$, and initiation of the mitochondria-dependent apoptotic pathway [19].

In a previous study conducted by us using a leukemic cell line, we identified lamin-A/C isoform 3, talin-1, and annexin A1 as universal biomarkers for PAH exposure [20]. Bone marrow derived mesenchymal stem cells (BM-MSCs), unlike leukemic cells, represent normal physiological conditions of the hematopoietic niche. There has been no comprehensive study to date, investigating the effect of FR on the integrity and function of BM-MSCs. Therefore, the aim of our study was to investigate the spectrum of cellular damage caused by FR and potential rescue effects of AHR antagonists and to identify biomarkers for exposure of BM-MSCs to FR.

\section{MATERIALS AND METHODS}

\section{Cell line and cell culture}

Bone marrow derived BM-MSCs were isolated and cultured using standard protocols [21]. For in vitro cell line studies, cells were cultured and maintained in Dulbecco's Modified Eagle Medium (DMEM) supplemented with 10\% fetal bovine serum and different concentrations of FR (25, $50,100,150$, and $200 \mu \mathrm{M})$. The medium was replaced every 24 hours to avoid chemical decay of FR.

\section{Reagents and antibodies}

FR (98\%) was purchased from Sigma (Sigma-Aldrich, St. Louis, MO, USA) and dissolved in dimethyl sulfoxide
(DMSO) (Sigma-Aldrich) to produce a stock solution at a concentration of $100 \mathrm{mM}$. A working solution was obtained with dilution of the stock solution using cell culture medium.

\section{Cell viability measurement}

For the assay of viable cell numbers, $2 \times 10^{3}, 4 \times 10^{3}$, and $5 \times 10^{3}$ cells per well were seeded in triplicate into a 96 well plate and subjected to an Enhanced Cell Viability Assay Kit (EZ-CyTox, Daeil Lab Service Co., Seoul, Korea), according to the instructions of the manufacturer. The tetrazolium-based colorimetric assay evaluates viable cell numbers, taking advantage of NADPH-dependent cellular oxidoreductase enzymes in viable cells to reduce the tetrazolium dye 3-(4,5-dimethylthiazol-2-yl)-2,5-diphenyltetrazolium bromide to insoluble formazan, which has a purple color. The absorbance at $450 \mathrm{~nm}$ of each well was measured using a VERSA Max microplate reader (Molecular Devices, Sunnyvale, CA, USA).

\section{DNA fragmentation assay}

DNA fragmentation assays were performed with the DNeasy mini kit (Qiagen, Valencia, CA, USA) according to the manufacturer's protocol. MSC cells were treated with $150 \mu \mathrm{M}$ and $200 \mu \mathrm{M}$ FR for 72 hours, after which genomic DNA was extracted. Next, DNA fragments were separated by gel electrophoresis on a $1.5 \%$ agarose gel. DNA was visualized using ethidium bromide staining and photographed under ultraviolet light.

\section{Detection of mitochondrial membrane potential}

Mitochondrial membrane potential was measured using MitoTracker Red CMXRos (Molecular Probes Inc., Eugene, OR, USA) according to the instructions provided by the manufacturer. Briefly, after 72 hours of treatment, MSC cells were washed twice with phosphate buffer saline (PBS) and loaded with $100 \mathrm{~nm}$ pre-warmed CMXRos for 30 minutes in serum free culture medium. Subsequently, cells were washed twice with fresh medium and transferred to a mounting chamber on the microscope stage. Images of red MitoTracker Red CMXRos fluorescence were captured using a 568-nm excitation light from the argon/krypton laser, a $560-\mathrm{nm}$ dichroic mirror, and a 590-nm long pass filter.

\section{Western blot analysis}

Protein expression was determined in BM-MSCs using the western blotting technique according to standard procedures. Briefly, total protein from untreated or treated cells was extracted in RIPA lysis buffer, and an equivalent amount of protein $(50 \mu \mathrm{g})$ from each group was separated with sodium dodecyl sulfate polyacrylamide gel electrophoresis (SDS-PAGE) and transferred onto a polyvinylidene fluoride (PVDF) membrane (Bio-Rad Laboratories, Hercules, CA, USA). After blocking with sodium azide at room temperature for 90 minutes, each membrane was incubated with a specific primary antibody $(1: 1,000)$ at $4^{\circ} \mathrm{C}$ overnight. After three washes in washing buffer $(20 \mathrm{mM}$ Tris- $\mathrm{HCl}, 500 \mathrm{mM}$ $\mathrm{NaCl}$, and $0.1 \%$ Tween 20 ), each membrane was incubated 
with the appropriate secondary antibody at room temperature for 90 minutes. Specific protein bands were visualized using a chemiluminescence detection system (Amersham ECL system, London, UK).

\section{Cellular proteome analysis}

Proteomic analysis was performed by a commercial laboratory (Yonsei Proteome Research Center, Seoul, Korea; www.proteomix.org). Briefly, the FR-treated cultured cells $\left(5 \times 10^{6} / \mathrm{mL}\right)$ were washed extensively with ice-cold PBS. The cells were then centrifuged at $600 \times \mathrm{G}$ for 5 minutes at $4^{\circ} \mathrm{C}$. The cells were resuspended in $1.0 \mathrm{~mL}$ of $1 \times$ cytosol extraction buffer containing dithiothreitol and protease inhibitors from a commercial mitochondria/cytosol fractionation kit (Pierce Biotechnology). Purification of the cytosolic fraction was conducted, and samples were prepared for and run on two-dimensional gel electrophoresis (2-DE), which was performed using previously published protocols [22]. Coomassie blue and silver-stained gels were digitized using a densitometer (Image Scanner) and analyzed with PD Quest 7.3.1 computer software (Bio-Rad). Using PD Quest tools, protein spots were enumerated, quantified, and characterized with respect to their molecular mass and isoelectric point by bilinear interpolation between the landmark features on each of the images previously calibrated with internal 2-DE standards (Bio-Rad).

All mass spectra were internally and externally calibrated using autoproteolytic trypsin fragments and a standard peptide mixture (Sigma-Aldrich). The mono-isotopic peptide mass fingerprinting data obtained from matrix-assisted laser desorption/ionization time-of-flight (MALDI-TOF) analysis were used to search for protein candidates using MS-Fit (http://prospector.ucsf.edu), Profound (http://www.prowl. rockefeller.edu), and Mascot (http://www.matrixscience.com) software programs. The searches were performed against SWISS-PROT/TrEMBL (http://www.expasy.ch/sprot) and National Center for Biotechnology Information (NCBI) (http://www.ncbi.nlm.nih.gov) databases. The identifications were accepted as positive when at least five matching peptides and at least $20 \%$ of the peptide coverage of the theoretical sequences matched within a mass accuracy of 50 or $25 \mathrm{ppm}$ with the internal calibration. Tryptic autolytic fragments and other contaminations were removed from the dataset used for the database search. In the MALDI-TOF/TOF analysis, the aminoacidic sequence tag obtained from each peptide fragmentation was used for protein identification in the searches of the SWISS-PROT/TrEMBL database. Proteomic analyses of mitochondria after FR exposure were additionally replicated three times.

\section{Flow cytometry}

For detection of apoptosis, treated and untreated MSC cells $\left(1 \times 10^{6} /\right.$ plate $)$ were stained with annexin $\mathrm{V}$-conjugated to fluorescein isothiocyanate (FITC) and propidium iodide (PI) using the annexin-V-FITC staining kit (BD Bioscience, San Jose, CA, USA) according to the manufacturer's instructions. Briefly, cells were suspended in $400 \mu \mathrm{L}$ of annexin $\mathrm{V}$ binding buffer and incubated with $3 \mu \mathrm{L}$ of annexin $\mathrm{V}$ and $3 \mu \mathrm{L}$ of PI for 15 minutes at room temperature in the dark. Images of at least 10,000 cells were acquired in a Beckman Coulter's Navios Tetra system using Kaluza analysis software. Percentages of cells undergoing apoptosis were determined by dual-color analysis. This staining allowed us to distinguish three subsets of cells: viable cells (annexin $\mathrm{V}$ negative and PI negative), early apoptotic cells (annexin $\mathrm{V}$ positive and PI negative), late apoptotic cells (annexin $\mathrm{V}$ positive and PI positive) and necrotic cells (annexin V negative and PI positive). Immediately after staining, the cells were analyzed in a flow cytometer using 488-nm excitation and a 525-nm band pass filter for FITC and a 620-nm filter for PI detection.

\section{RESULTS}

\section{Change in total cell numbers and morphology}

Cell morphology of BM-MSCs was visually observed under a microscope every 24 hours for three days after treating with FR and the AHR antagonist CH223191. The morphology in these three days showed a similar pattern as compared to the total cell number, which was remarkably decreased with FR treatment. However, co-culture with CH223191 rescued the loss of cell number in a dose-dependent manner. After FR treatment, cells were elongated, detached from the subsurface of the culture plate, showed a spindle-like form, and had loosened cell-to-cell attachment compared to the control group, which were cobble-stone shaped and tightly attached to each other. In contrast, a rescue effect in the CH223191 treated cells was revealed by the increase in cell number and retrieval of cell morphology similar to that of the control group (Fig. 1B).

\section{Cell viability}

The tetrazolium-based colorimetric assay was used to evaluate the cytotoxicity of FR treatment on the BM-MSCs by measuring cell viability. BM-MSCs $\left(5 \times 10^{3}, 4 \times 10^{3}\right.$, and $2 \times 10^{3}$ cells/well) were treated with $50,100,150$, and $200 \mu \mathrm{M}$ concentrations of FR for 72 hours. Cell viability gradually decreased with increase of FR dosage. The $\mathrm{IC}_{50}$ of FR for BM-MSCs was approximately $50 \mu \mathrm{M}$; therefore, FR concentrations above the $\mathrm{IC}_{50}$ significantly induced decreases in MSC numbers (Fig. 2A).

\section{Apoptosis and mitochondrial membrane potential}

To determine whether FR induces apoptosis in MSC cells, changes in the nuclear DNA, plasma membrane, and organelles, especially the mitochondria, were checked by DNA fragmentation assay, annexin V-PI-based apoptosis assay, and the MitoTracker Red CMXRos fluorescence dye assay, respectively (Fig. 2B-D). Compared with DMSO control, treatment with $150 \mu \mathrm{M}$ and $200 \mu \mathrm{M}$ FR concentrations resulted in almost double the number of cells undergoing early apoptosis (Fig. 2C). However, clear single bands in the DNA fragmentation assay indicates that nuclear DNA was still intact in the FR treated cells (Fig. 2B). Expression of caspase 


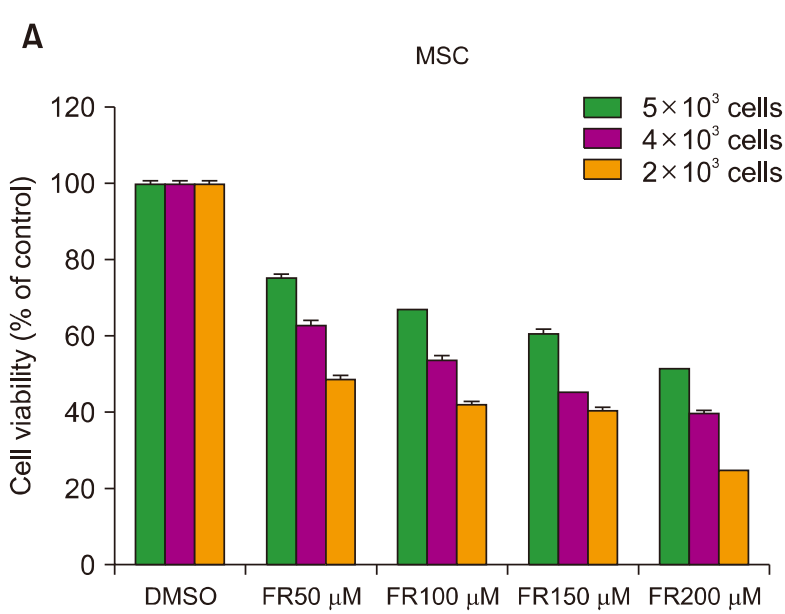

C
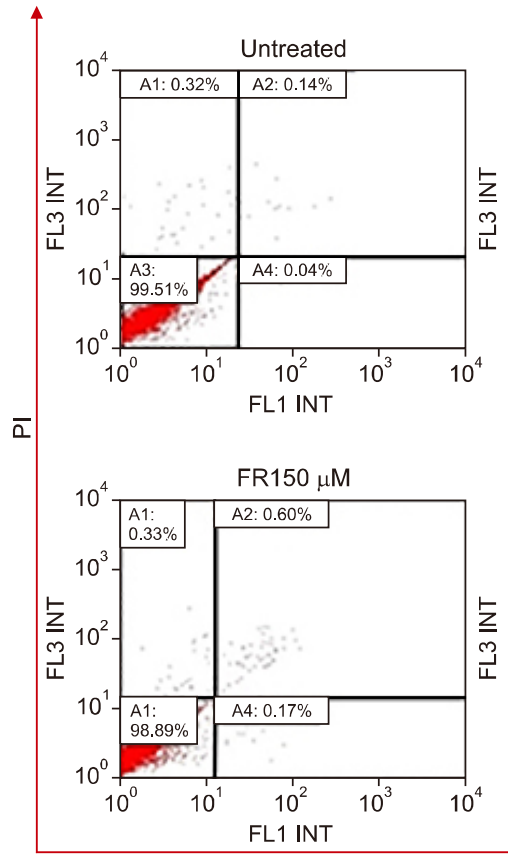

FITC-annexin V

B

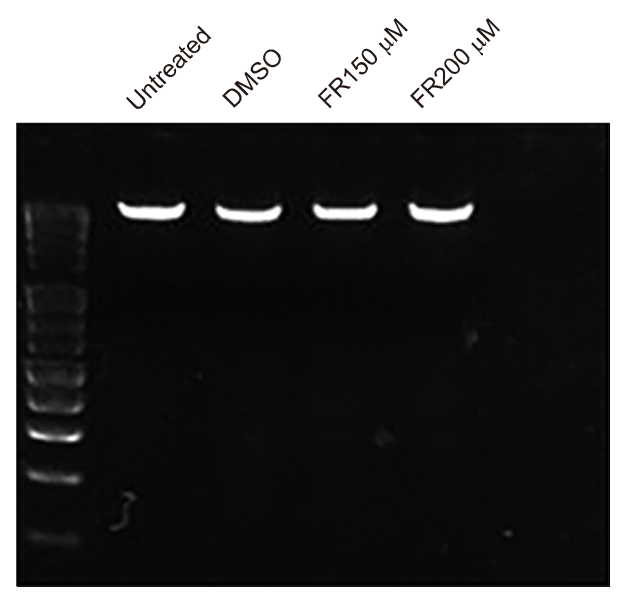

D

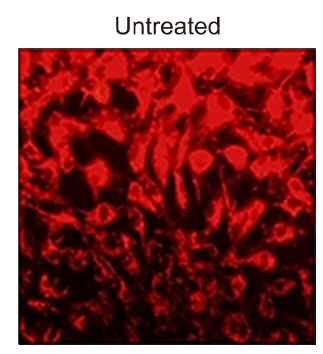

$\mathrm{FR} 150 \mu \mathrm{M}$

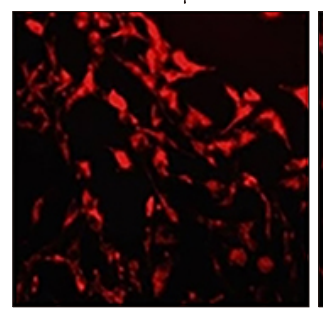

$0.1 \%$ DMSO

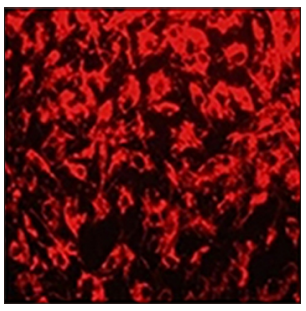

$\mathrm{FR} 200 \mu \mathrm{M}$

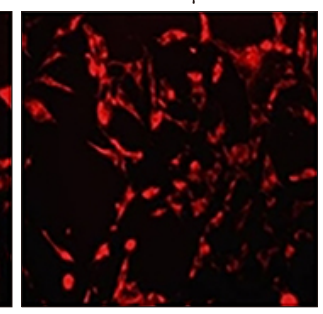

Fig. 2. Cytotoxic effect of FR on BM-MSCs. MSCs $\left(5 \times 10^{3}, 4 \times 10^{3}\right.$, and $2 \times 10^{3}$ cells/well) were treated with indicated concentrations (50, 100, 150, and $200 \mu \mathrm{M})$ of FR for 72 hours. Cell viability was measured by the tetrazolium-based colorimetric assay. Cell viability gradually decreased with increased FR dosage. The IC 50 of FR was $50 \mu \mathrm{M}$ (A). DNA fragmentation assay was performed in MSCs after treatment with FR for 72 hours. There were no significant changes in the nuclear DNA in response to FR (B). Effect of FR in inducing apoptosis in MSCs. Cells $\left(1 \times 10^{6} /\right.$ plate) treated with $150 \mu \mathrm{M}$ and $200 \mu \mathrm{M}$ FR for 72 hours were co-stained with PI and FITC-conjugated annexin V and then examined by flow cytometry. Almost twice as many cells underwent apoptosis after FR treatment compared to control (C). Effect of FR exposure on mitochondria in MSCs. Loss of mitochondrial membrane potential was observed after FR treatment. MSCs were treated with FR for 72 hours, washed twice with phosphate buffer saline (PBS), and then treated with $100 \mathrm{~nm}$ MitoTracker Red CMXRos at $37^{\circ} \mathrm{C}$ for 30 minutes. Fluorescence images were then recorded. Reduced fluorescence with increasing doses of FR is an indication of mitochondrial membrane potential loss, which is an indication of mitochondrial dysfunction as well as initiation of early apoptosis (D).

3 and caspase 9 increased and the anti-apoptotic protein bcl-2 decreased in a dose-dependent manner with increasing concentrations of FR, indicating apoptosis activation (Fig. 3A).

Since MitoTracker Red CMXRos probe contains a mildly thiol-reactive chloromethyl moiety, this dye passively diffuses across the plasma membrane and accumulates in active mitochondria. Therefore, decreased fluorescence is indicative of mitochondrial dysfunction and initiation of cell apoptosis. Mitochondrial membrane potential of BM-MSCs after FR exposure was remarkably decreased above concentrations of $150 \mu \mathrm{M}$ (Fig. 2D). 

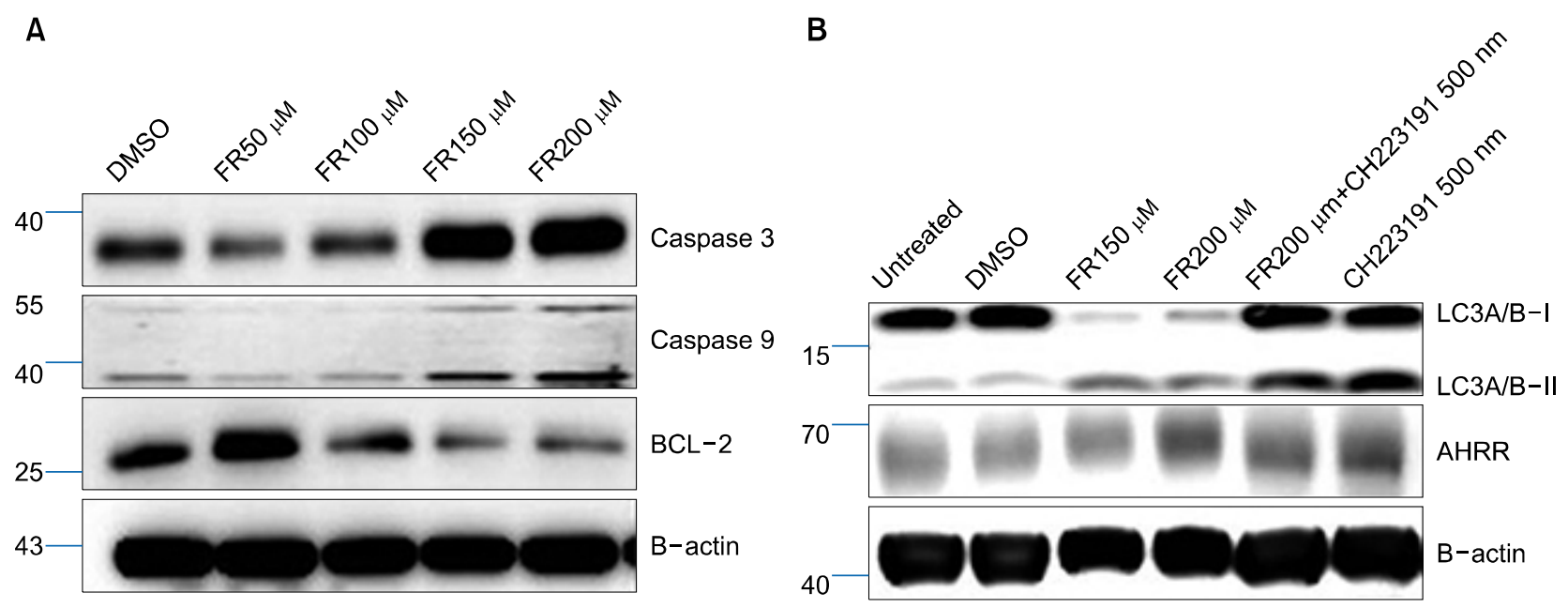

Fig. 3. Molecular signaling pathway of apoptosis and autophagy with FR exposure and an AHR inhibitor by western blot. Expression of caspase 3 and caspase 9 increased, and the anti-apoptotic protein bcl-2 decreased in a dose-dependent manner with increasing concentrations of FR, which is an indication of apoptosis activation (A). FR $(150 \mu \mathrm{M}$ and $200 \mu \mathrm{M})$ induced conversion of LC3A I to LC3A II, which confirms induction of autophagy, which was recovered by treatment with the AHR antagonist $\mathrm{CH} 223191$ (B).

\section{Rescue effect of AHR antagonist}

AHR is a ligand-activated transcription factor involved in the regulation of biological responses to PAHs. Thus, FR induces cytotoxicity via binding to AHR. In this study, several AHR antagonists were screened and selected from the library established by the Korea Chemical Bank (http:// eng.chembank.org). Among several AHR antagonists, CH223191 was selected from our preliminary tests for AHR blockage experiments. Co-treatment of CH223191 with FR rescued cell viability and morphology. These findings indicate that the AHR antagonist CH223191 has the potential to protect the cells and rescue FR-induced cytotoxicity (Fig. 1B).

\section{Alteration of autophagy activity}

The expression of LC3A I and LC3A II is an indication of autophagy induction and was observed at 150 and 200 $\mu \mathrm{M}$ FR concentrations (Fig. 3B). During autophagy, LC3-I is converted to LC3-II through lipidation by a ubiquitin-like system involving Atg7 and Atg3, thus allowing LC3 to become coupled with autophagic vesicles. The presence of LC3 in autophagosomes and conversion of LC3-I to LC3-II have been used as indicators of autophagy.

\section{Identification of biomarkers for FR exposure}

For proteomic analysis, cytosol fractions from FR-treated and DMSO-treated BM-MSCs were sent to a well-established commercial proteomic laboratory. After image analysis on 2-DE gels, a total of 48 spots were analyzed (26 upregulated and 22 downregulated proteins) (Fig. 4). Peptide mass fingerprinting was performed with MALDI-TOF on in-gel trypsin-digested proteins for the identification of aberrantly expressed proteins. Initially, four proteins (annexin A6, pyruvate kinase, UDP-glucose dehydrogenase, and phospholipase A2) were up regulated in the FR samples, with more than 4-fold increases compared to the controls. One protein
(PR264) decreased aberrantly (more than 4-fold) in the FR exposure group compared to that in the controls (Table 1).

\section{DISCUSSION}

This study demonstrated that direct exposure of FR for 72 hours in BM-MSCs induced cellular damage and that the AHR antagonist CH223191 has protective effect against FR toxicity. Cells compromised by FR activate apoptosis and autophagy pathways. Furthermore, Annexin A6, pyruvate kinase, UDP-glucose dehydrogenase, and phospholipase A2 could be potential biomarkers for FR exposure.

Decreases in cell counts after FR treatment raises the possibility that FR induces apoptosis in MSC cells. To test this possibility, we examined apoptosis-related proteins, mitochondrial membrane potential $\left(\Delta \psi_{\mathrm{m}}\right)$, nuclear DNA fragmentation, and plasma membrane integrity after treatment with FR. Our study revealed that $\Delta \psi_{\mathrm{m}}$ decreased significantly in a dose-dependent manner while nuclear DNA remained intact. Previous studies have shown that nuclear signs of apoptosis follow the disruption of the $\Delta \psi_{\mathrm{m}}$ in a variety of different systems [23-25]. Loss of mitochondrial membrane potential is an indication of early apoptosis and it fulfills the following criteria: 1) precocity in the sense that it precedes all other manifestation of apoptosis, including plasma membrane alterations, chromatin condensation, and DNA fragmentation; 2) specificity in the sense that it is only observed in pre-apoptotic cells; 3) universality in the sense that it is found in all cell types undergoing apoptosis and in response to all apoptosis inducers investigated; and 4) irreversibility in the sense that it marks a point-of-no-return, and that cells which have their $\Delta \psi_{\mathrm{m}}$ disrupted will undergo apoptosis even when the apoptosis-inducing stimulus is withdrawn [23-25]. Altogether, these characteristics underline 


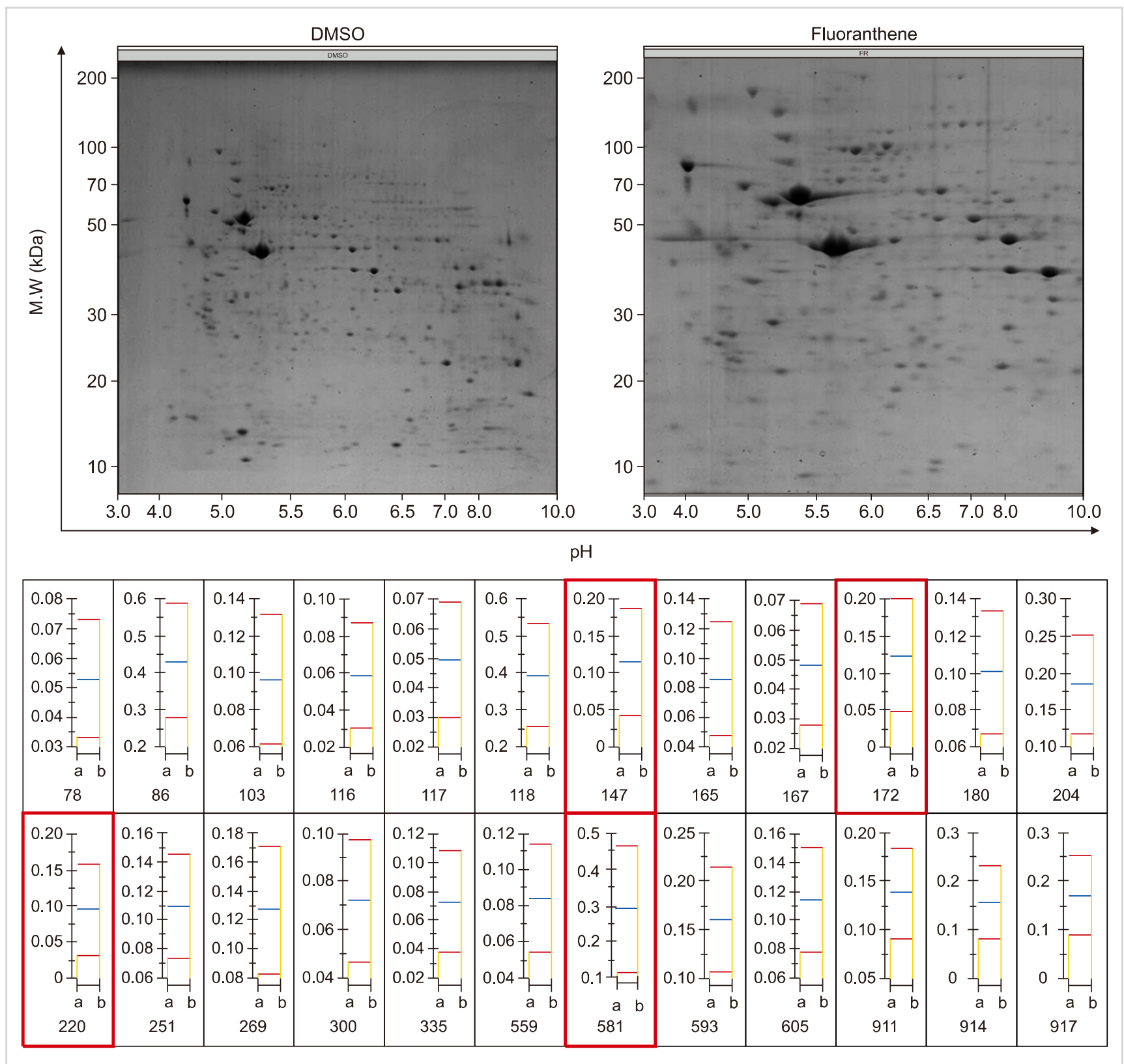

Fig. 4. Proteomic analysis for the identification of candidate biomarkers for FR exposure. Two-dimensional electrophoresis showed altered expression of various protein spots (upper panel), which are quantified as bar graphs (lower panel) of relative quantitative expression of each candidate proteomic marker. Four altered spots $(147,172,220$, and 581, red boxes) were identified as annexin A6, pyruvate kinase protein, UDP-glucose dehydrogenase, and phospholipase A2.

Table 1. Identification of candidate biomarkers for the exposure of fluoranthene.

\begin{tabular}{lccc}
\hline \multicolumn{1}{c}{ Biomarkers } & $\begin{array}{c}\text { DMSO, } \\
\% \text { vol }\end{array}$ & $\begin{array}{c}\mathrm{FR}, \\
\% \text { vol }\end{array}$ & $\begin{array}{c}\text { FR/DMSO, } \\
\text { ratio }\end{array}$ \\
\hline Annexin A6, isoform CRA & 0.043 & 0.189 & 4.4 (increase) \\
Pyruvate kinase protein & 0.047 & 0.199 & 4.2 (increase) \\
UDP-glucose dehydrogenase & 0.028 & 0.159 & 5.6 (increase) \\
Phospholipase A2 & 0.397 & 0.095 & 4.2 (increase) \\
PR 264 & 0.112 & 0.467 & 4.2 (decrease) \\
\hline
\end{tabular}

the utility of $\Delta \psi_{\mathrm{m}}$ measurements for the characterization of early apoptosis.

However, treatment with the AHR antagonist CH223191 showed a dose-dependent cellular rescue effect from FR damage in this study. A previous study showed that CH223191 exerts its antagonist effect against TCDD through its ability to act as a competitive ligand [26], and this is demonstrated through both ligand-binding analysis [27] and the increase in agonist activity (i.e. AHR-dependent DNA binding and gene expression) which occurs with increasing concentrations of TCDD in the presence of a constant concentration of CH223191. 
FR exposure increased expression of hnRNP and decreased expression of lamin A/C in MSCs. In a previous study [20], we investigated the profiling of polycyclic aromatic hydrocarbon-(PAH)-induced genotoxicity in several cell lines and in zebrafish. In that study, each type of cell displayed different proportionalities of apoptosis. Mitochondrial DNA (mtDNA) copy number was dramatically elevated after 5-day treatment of fluoranthene and pyrene. The notably deregulated proteins following $\mathrm{PAHs}$ exposure were: lamin-A/C isoform 3 and annexin A1 for benzopyrene; lamin-A/C isoform 3 and DNA topoisomerase 2-alpha for pentacene; poly[ADP-ribose] polymerase 1 (PARP-1) for fluoranthene; talin-1 and DNA topoisomerase 2-alpha for pyrene. We also showed that the expression of an AHR repressor was increased, which indicated the occurrence of natural defense mechanisms in BM-MSCs. We found that FR induced expression of autophagy proteins, which is a typical cellular response to nutrient deprivation [28]. However, AHR antagonist CH223191 was able to rescue this cellular stress. Further experiments are needed to elucidate the underlying cellular mechanism. There were significant changes in the apoptosis related proteins, which induced the activation of apoptosis-related molecular signaling pathways in BM-MSCs after even exposure to FR. Our flow cytometry results also support this observation, as it was observed that almost twice as many cells underwent early apoptosis in FR treated cells compared to the control group.

After FR treatment, five deregulated proteins stood out in our proteomics study. We can infer possible mechanisms from these proteins, even though further research is needed. After exposure to FR for several days there is a tug-of-war between cellular survival and cellular apoptosis. Annexin A6 participates in plasma membrane repair and impacts receptor signaling at the cell surface, growth factors, lipoprotein receptor trafficking, $\mathrm{Ca}^{2+}$ channel activity, and $\mathrm{T}$ cell activation [29]. Expression of several kinase proteins (e.g. pyruvate kinase and UDP-glucose dehydrogenase) in metabolic pathways increased to provide sufficient energy to combat the toxic threat. Phospholipase A2 plays a modifying role in the apoptotic cell death process [30].

In summary, direct exposure of BM-MSCs to FR for 72 hours induced cellular damage. Cells express an arsenal of proteins to combat the exogenous attack. Cells compromised by FR activate apoptosis and autophagy pathways. However, the AHR antagonist CH223191 has a protective effect against FR toxicity, which has implications for the development of drugs for clinical use to prevent FR toxicity in bone marrow derived BM-MSCs. Furthermore, Annexin A6, pyruvate kinase, UDP-glucose dehydrogenase, and phospholipase A2 could be potential biomarkers for FR exposure to bone marrow derived BM-MSCs.

\section{Authors' Disclosures of Potential Conflicts of Interest}

No potential conflicts of interest relevant to this article were reported.

\section{REFERENCES}

1. Raychoudhury SS, Kubinski D. Polycyclic aromatic hydrocarboninduced cytotoxicity in cultured rat Sertoli cells involves differential apoptotic response. Environ Health Perspect 2003;111:33-8.

2. Cherng SH, Lin ST, Lee H. Modulatory effects of polycyclic aromatic hydrocarbons on the mutagenicity of 1-nitropyrene: a structure-activity relationship study. Mutat Res 1996;367:177-85.

3. Lewtas J, Walsh D, Williams R, Dobiás L. Air pollution exposureDNA adduct dosimetry in humans and rodents: evidence for non-linearity at high doses. Mutat Res 1997;378:51-63.

4. Zedeck MS. Polycyclic aromatic hydrocarbons: a review. J Environ Pathol Toxicol Oncol 1980;3:537-67.

5. Busbee DL, Norman JO, Ziprin RL. Comparative uptake, vascular transport, and cellular internalization of aflatoxin-B1 and benzo(a)pyrene. Arch Toxicol 1990;64:285-90.

6. Monteith DK, Novotny A, Michalopoulos G, Strom SC. Metabolism of benzo[a]pyrene in primary cultures of human hepatocytes: dose-response over a four-log range. Carcinogenesis 1987;8:983-8.

7. Kiefer F, Cumpelik O, Wiebel FJ. Metabolism and cytotoxicity of benzo(a)pyrene in the human lung tumour cell line NCI-H322. Xenobiotica 1988;18:747-55.

8. McKinney PA, Fear NT, Stockton D; UK Childhood Cancer Study Investigators. Parental occupation at periconception: findings from the United Kingdom Childhood Cancer Study. Occup Environ Med 2003;60:901-9.

9. Castro-Jiménez MÁ, Orozco-Vargas LC. Parental exposure to carcinogens and risk for childhood acute lymphoblastic leukemia, Colombia, 2000-2005. Prev Chronic Dis 2011;8:A106.

10. Chang JS. Parental smoking and childhood leukemia. Methods Mol Biol 2009;472:103-37.

11. Milne E, Greenop KR, Scott RJ, et al. Parental prenatal smoking and risk of childhood acute lymphoblastic leukemia. Am J Epidemiol 2012;175:43-53.

12. Visser O, van Wijnen JH, van Leeuwen FE. Residential traffic density and cancer incidence in Amsterdam, 1989-1997. Cancer Causes Control 2004;15:331-9.

13. Pearson RL, Wachtel H, Ebi KL. Distance-weighted traffic density in proximity to a home is a risk factor for leukemia and other childhood cancers. J Air Waste Manag Assoc 2000;50:175-80.

14. Griesbaum K, Behr A, Biedenkapp D, et al. Hydrocarbons. In: Elvers B, ed. Ullmann's encyclopedia of industrial chemistry. 7th ed. Weinheim, Germany: Wiley-VCH, 2011.

15. Busby WF Jr, Goldman ME, Newberne PM, Wogan GN. Tumorigenicity of fluoranthene in a newborn mouse lung adenoma bioassay. Carcinogenesis 1984;5:1311-6.

16. Zhu H, Li Y, Trush MA. Characterization of benzo[a]pyrene quinone-induced toxicity to primary cultured bone marrow stromal cells from DBA/2 mice: potential role of mitochondrial dysfunction. Toxicol Appl Pharmacol 1995;130:108-20.

17. Li N, Sioutas $\mathrm{C}$, Cho A, et al. Ultrafine particulate pollutants induce oxidative stress and mitochondrial damage. Environ Health Perspect 2003;111:455-60.

18. Xia T, Korge P, Weiss JN, et al. Quinones and aromatic chemical compounds in particulate matter induce mitochondrial dysfunction: implications for ultrafine particle toxicity. Environ Health 
Perspect 2004;112:1347-58.

19. Ko CB, Kim SJ, Park C, et al. Benzo(a)pyrene-induced apoptotic death of mouse hepatoma Hepa1c1c7 cells via activation of intrinsic caspase cascade and mitochondrial dysfunction. Toxicology 2004;199:35-46.

20. Kim HY, Kim HR, Kang MG, et al. Profiling of biomarkers for the exposure of polycyclic aromatic hydrocarbons: lamin-A/C isoform 3 , poly[ADP-ribose] polymerase 1 , and mitochondria copy number are identified as universal biomarkers. Biomed Res Int 2014;2014:605135.

21. Gnecchi M, Melo LG. Bone marrow-derived mesenchymal stem cells: isolation, expansion, characterization, viral transduction, and production of conditioned medium. Methods Mol Biol 2009;482:281-94.

22. Xie J, Techritz S, Haebel S, et al. A two-dimensional electrophoretic map of human mitochondrial proteins from immortalized lymphoblastoid cell lines: a prerequisite to study mitochondrial disorders in patients. Proteomics 2005;5:2981-99.

23. Cossarizza A, Franceschi C, Monti D, et al. Protective effect of $\mathrm{N}$-acetylcysteine in tumor necrosis factor-alpha-induced apoptosis in U937 cells: the role of mitochondria. Exp Cell Res 1995;220: 232-40.

24. Castedo M, Macho A, Zamzami N, et al. Mitochondrial perturbations define lymphocytes undergoing apoptotic depletion in vivo. Eur J Immunol 1995;25:3277-84.

25. Marchetti P, Susin SA, Decaudin D, et al. Apoptosis-associated derangement of mitochondrial function in cells lacking mitochondrial DNA. Cancer Res 1996;56:2033-8.

26. Zhao B, Degroot DE, Hayashi A, He G, Denison MS. CH223191 is a ligand-selective antagonist of the Ah (Dioxin) receptor. Toxicol Sci 2010;117:393-403.

27. Kim SH, Henry EC, Kim DK, et al. Novel compound 2-methyl2H-pyrazole-3-carboxylic acid (2-methyl-4-o-tolylazo-phenyl)amide (CH-223191) prevents 2,3,7,8-TCDD-induced toxicity by antagonizing the aryl hydrocarbon receptor. Mol Pharmacol 2006;69:1871-8.

28. Poot M, Zhang YZ, Krämer JA, et al. Analysis of mitochondrial morphology and function with novel fixable fluorescent stains. J Histochem Cytochem 1996;44:1363-72.

29. Cornely R, Rentero C, Enrich C, Grewal T, Gaus K. Annexin A6 is an organizer of membrane microdomains to regulate receptor localization and signalling. IUBMB Life 2011;63:1009-17.

30. Atsumi G, Tajima M, Hadano A, Nakatani Y, Murakami M, Kudo I. Fas-induced arachidonic acid release is mediated by $\mathrm{Ca} 2+-$ independent phospholipase A2 but not cytosolic phospholipase A2, which undergoes proteolytic inactivation. J Biol Chem 1998;273:13870-7. 